\title{
On-Demand Grid Application Tuning and Debugging with the NetLogger Activation Service
}

\author{
Dan Gunter, Brian L. Tierney, Craig E. Tull, Vibha Virmani \\ Lawrence Berkeley National Laboratory \\ \{dkgunter,bltierney,cetull,vvirmani\}@lbl.gov
}

\begin{abstract}
Typical Grid computing scenarios involve many distributed hardware and software components. The more components that are involved, the more likely it is that one of them may fail. In order for Grid computing to succeed, there must be a simple mechanism to determine which component failed and why. Instrumentation of all Grid applications and middleware is an important part of the solution to this problem. However, it must be possible to control and adapt the amount of instrumentation data produced in order to not be flooded by this data. In this paper we describe a scalable, high-performance instrumentation activation mechanism that addresses this problem.
\end{abstract}

\section{Introduction}

Grid monitoring is the measurement and publication of the state of a Grid component at a particular point in time. To be effective, monitoring must be "end-to-end", meaning that all components between the application endpoints must be monitored. This includes software (e.g., applications, services, middleware, operating systems), end-host hardware (e.g., CPUs, disks, memory, network interface), and networks (e.g., routers, switches, or end-to-end paths).

Monitoring is required for a number of purposes, including status checking, troubleshooting, performance tuning, and debugging. For example, assume a Grid job which normally takes 15 minutes to complete has been running for two hours but has not yet completed. Determining what, if anything, is wrong is difficult and requires a great deal of monitoring data. Is the job still running or did one of the software components crash? Is the network congested? Is the CPU loaded? Is there a disk problem? Was a software library containing a bug installed somewhere? Monitoring provides the information to help track down the current status of the job and locate any problems.
A complete end-to-end Grid monitoring system has several components, including:

Instrumentation: Instrumentation is the process of putting probes into software or hardware to measure the state of a hardware component, such as a host, disk, network, or a software component, such as operating system, middleware, or application. These probes are often called Sensors.

Monitoring Event Publication: Consumers of monitoring event data need to locate appropriate monitoring event providers. Standard schemas, publication mechanisms, and access policies for monitoring event data are required.

Event Archives: Archived monitoring event data is critical for performance analysis and tuning, as well as for accounting. Historical data can be used to establish a baseline upon which to compare current performance.

Sensor Management: As Grid environments become bigger and more complex, there are more components to monitor and manage, and the amount of monitoring data produced by this effort can quickly become overwhelming. Some components require constant monitoring, while others only are monitored on demand. A mechanism for activating sensors on demand is required.

In this paper we describe a Grid activation service which is designed to address the problem of starting, stopping, and changing the level of instrumentation data in running Grid processes. This is done in a manner that is completely transparent to the application. The activation service is designed to work in a cluster environment, and be efficient and scalable. The activation service also collects the instrumentation results, and forwards them to all interested consumers of this data.

This activation service is built using components from NetLogger [16] and pyGMA. pyGMA is our implementation of the Global Grid Forum Grid Monitoring Architecture [15]. NetLogger is used to instrument Grid applications and services, and includes the ability to change the logging-level on the fly by 
periodically examining a configuration file [7]. The NetLogger binary data format provides an extremely efficient, light-weight transport mechanism for the monitoring data. pyGMA provides an easy to use, SOAP-based framework for control messages. pyGMA also provides a standard publish-subscribe API for Grid monitoring event publication.

\subsection{The Activation Service and Athena}

An example application use-case for the Activation Service is Athena. The Athena [1] object-oriented framework is designed to provide a common infrastructure and environment for simulation, filtering, reconstruction and analysis applications for the current generation of high energy physics experiments.

Consider the problem of developing, tuning, and running the Atlas Athena Framework in a Grid Environment. The first step is to insert instrumentation code to ensure the program is operating as expected. This can be done using an instrumentation package such as NetLogger, and instrumentation code should be added to generate timestamped monitoring events before and after any CPU intensive tasks, and before and after all disk and network $\mathrm{I} / \mathrm{O}$, as is explained in [10].

Once the application is debugged and tested, it is ready for production use. Other monitoring services now become important. The level of instrumentation required for the debugging scenario above can easily generate thousands of monitoring events per second. Clearly one does not need or want this level of monitoring activated all the time, so some type of monitoring activation service is needed so that a user can turn instrumentation on and off in a running service.

Next, it is useful to establish a performance baseline for this service, and store this information in the monitoring event archive. System information such as processor type and speed, OS version, CPU load, disk load, and network load data should be collected during the baseline test runs. The monitoring event publication service is needed to locate the sensors and initiate a subscription for the resulting monitoring data. Several tests are then run, sending complete application instrumentation (for clients, servers, and middleware), host, and network monitoring data to the archive. A more detailed example is given in [9].

The components required for this scenario are shown in Figure 1. Athena jobs are running on nodes of one or more compute clusters. The user contacts a monitoring data registry to obtain the location of the activation service that is managing the instrumentation level and producing monitoring data for these Athena jobs. The user requests of the activation service that the instrumentation level be increased from the default level (e.g., just error conditions) to a higher level (e.g., full performance trace). The user

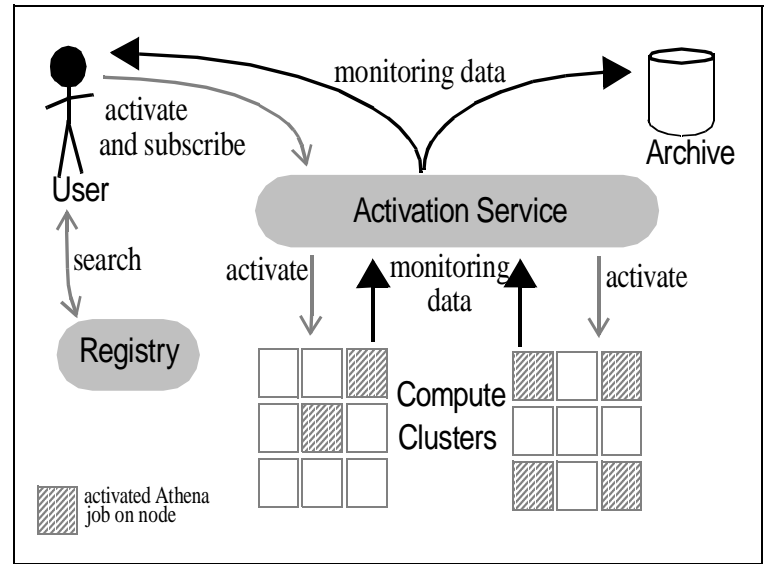

Figure 1: Sample Use of Activation Service

then subscribes for the instrumentation data, telling the activation service to send the data both to the monitoring archive and back to the user. The activation service collects the data from each of the cluster nodes, and forwards it to both the user and to the monitoring archive.

More details on each of these components are in Section 4., below.

\section{Related Work}

There are many monitoring systems out there, such as the Condor project's Hawkeye [8], which have publish/subscribe interfaces and some sort of filtering capabilities. However, these systems are not concerned with application instrumentation, low thresholds for intrusiveness, or mechanisms for instrumentation activation mechanisms. Kernel instrumentation packages such as MAGNeT [5] are extremely efficient, but often assume the data can be stored in memory until the program exits, and also may require kernel modifications. There are also automatic and semi-automatic application-level instrumentation systems such as Paradyn [11], which are efficient but have simple models for delivering the results and often are specialized for a particular programming model (e.g., parallel programming codes). Although all these systems share some goals with the activation service, none have the particular focus on efficient, general-purpose application instrumentation in a wide-area distributed setting.

The Open Grid Services Architecture (OGSA) [6] incorporates at a fundamental level much of the functionality required to implement a Grid monitoring service. Any OGSA Grid service can have associated with it arbitrary service data elements (SDEs): named and typed data objects that can be both queried and subscribed to by other parties. The Open Grid Services Infrastructure (OGSI) [18] specification provides specific behaviors for the notification interface outlined in the OGSA. 


\section{Building Blocks for the Activation Service}

The activation service is a higher-level Grid service based on the underlying components of GMA and NetLogger, described in this section.

\subsection{Grid Monitoring Architecture}

In 1999 a working group was formed within the Global Grid Forum with the goal of defining a scalable architecture for Grid monitoring. This group has produced both a set of requirements for Grid monitoring, and a high-level specification for a Grid Monitoring Architecture (GMA). The Activation Service's publication interface is based upon this architecture.

In GMA, the basic unit of monitoring data is called an event. An event is a named, timestamped, structure that may contain one or more items of data. This data may relate to one or more resources such as memory or network usage, or be application-specific data like the amount of time it took to multiply two matrices. The component that makes the event data available is called a producer, and a component that requests or accepts event data is called a consumer. A directory service (a.k.a. registry) is used to publish what event data is available and which producer to contact to request it.

A producer and consumer can be combined to make what is called a producer/consumer pipe. This can be used to filter or aggregate data. For example, a consumer might collect event data from several producers, and then use that data to generate a new derived event data type, which is then made available to other consumers. More elaborate filtering, forwarding, and caching behaviors could be implemented by connecting multiple consumer/producer pipes. The activation service described in this paper is an example of a GMA producer/consumer pipe.

A number of groups are now developing monitoring services based on the GMA architecture, such as R-GMA [3] (Relational GMA, so-called because it uses a relational model for all data) ReMoS [4], and TOPOMON [2].

The OGSA notification service is very similar to GMA. The OGSA interface specifies a notification source and sink, which are very similar to a producer and consumer in the GMA. However the current OGSI specification does not provide an unsubscribe operation, or specify a subscription language, and the notification sink requires that all messages be XML. Integration with alternate transport methods for monitoring data such as NetLogger or BEEP [14] will require more support from the OGSI specification.

\section{2 pyGMA}

The pyGMA [13], for "Python GMA", is our implementation of the Grid Monitoring Architecture (GMA) producer, consumer, and registry. It implements Web-Services SOAP interfaces in Python, a high-level object-oriented language. It uses SOAP to aid with serialization and deserialization of messages. Using the pyGMA, only a small amount of Python code would be needed to subscribe to a GMA producer (e.g., the Activation Producer, described below) for events, directing the results to be transported using NetLogger or query a producer for one or more events (returned directly in XML).

\subsection{NetLogger}

At Lawrence Berkeley National Lab we have developed the NetLogger Toolkit [17], which is designed to facilitate non-intrusive instrumentation of distributed computing components. Using NetLogger, distributed application components are modified to produce timestamped logs of "interesting" events at all the critical points of the distributed system. Events from each component are correlated, which allows one to characterize the performance of all aspects of the system and network in detail.

The NetLogger instrumentation library is very efficient and easy to use. Using the binary format, NetLogger can serialize on the order of half a million events per second [16]. In order to instrument an application to produce event logs, the application developer inserts calls to the NetLogger API at all the critical points in the code, then links the application with the NetLogger library. This facility is currently available in several languages: Java, C, $\mathrm{C}++$, Python, and Perl. The API has been kept as simple as possible, while still providing automatic timestamping of events and logging to either memory, a local file, syslog, a remote host. Sample Python NetLogger API usage is shown in Figure 2. As is shown in this example,

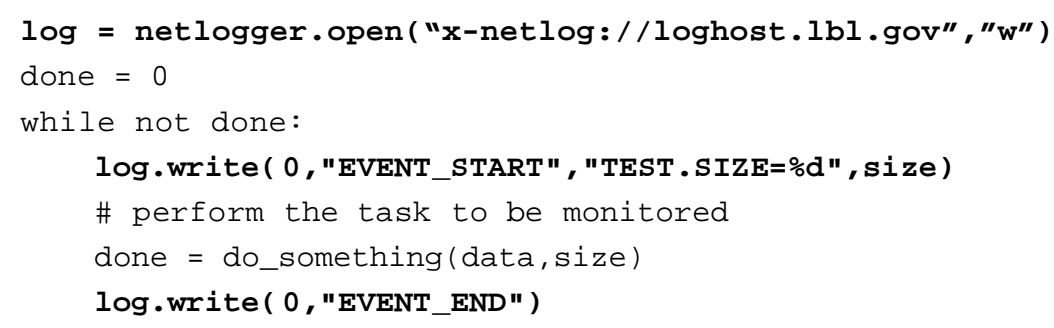

Figure 2: Sample NetLogger Usage 
"interesting" events in the code (such as I/O or processing) are typically wrapped with NetLogger write() calls that generate user-defined start and end instrumentation events

The NetLogger ASCII format consists of a whitespace-separated list of "field=value" pairs. Required fields are DATE, HOST, PROG, NL.EVENT and LVL; these can be followed by any number of user-defined fields. Here is a sample NetLogger event:

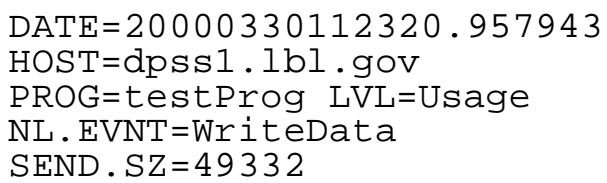

This says that the program testProg on host dpss1.lbl.gov sent 49322 bytes of data on March 30, 2000, 11:23am (and some seconds). NetLogger can generate XML formatted data as well. The NetLogger binary format is much faster, but harder for third-party tools to use. NetLogger includes tools for converting between the XML, ASCII, and binary formats.

\subsection{Grid Event Transport}

Typically, instrumentation systems only address the problem of extracting the data and writing it to memory or local disk. In a Grid environment, it is just as important to have a robust, efficient means for transporting the instrumentation data beyond that "first hop", to one or more consumers, each of whom may be interested in a different subset of the same instrumentation data. A transport to accomplish this needs to overcome several challenges. Opening connections across the WAN is expensive, so the transport should be able to stream an arbitrary amount of data across a network connection. Temporary network or server failures in the Grid are the rule, not the exception, so the transport must be reliable in the face of, e.g., broken TCP connections. Because pauses to write out instrumentation are rarely tolerable to the application, data should be buffered before every potential bottleneck (e.g., before anyWAN hop).

Finally, delivering a different subset of the data to different consumers requires applying filters on the data at intermediate nodes. To make this feasible on a Grid scale, we believe that the encoding rules and underlying data model should be part of the transport. The encoding offered should be efficient: the component being monitored cannot be perturbed, and intermediaries should be able to apply filters or analyze the data at close the rate the data is generated. In order to help make the encoding efficient, and also to simplify the task of creating and processing the data, the data model should be simple (i.e., not relational or XML-Infoset). Our approach is similar to that of the XML Metadata Integration Toolkit, (XMIT) from Georgia Tech [19].
NetLogger has been designed to answer these requirements of a Grid transport. It has the following features:

- Efficient streaming. NetLogger improves streaming efficiency by buffering all writes for up to $64 \mathrm{~K}$ or 1 second (whichever comes first).

- Reliability. The write API allows the user to specify a "backup" file. If a TCP connection fails, the log data is saved to the backup and, optionally, automatically sent over once that connection comes back up again.

- Buffering. The Activation Service directs all logging to local disk, and then reads from these disk buffers in order to forward the data to consumers.

- Efficient encoding. NetLogger supports a very efficient binary format, a fairly efficient, readable ASCII, format, and XML. The NetLogger API's can transparently handle each format.

- Minimal data model. Each logged item, or "event", is a timestamped set of typed name/value pairs.

The restricted NetLogger data model and binary encoding provide efficiency where it is needed; the general-purpose GMA publication architecture with XML-based SOAP messaging provides flexibility where it is needed. The cooperation of these technologies provides a scalable foundation for the Activation Service.

\section{Activation Service components}

The Activation Service has three main components: the Activation Node, the Activation Producer, the Activation Manager. When multiple activation services are deployed, a fourth component, the Registry, is also needed. These components are deployed as shown in Figure 3, with one activation node per host, one activation producer and manager per logical host group (e.g., a cluster), and only one logical registry for, ideally, the whole Grid. Distributed search technologies such as LDAP, peer-to-peer technologies, or reliable multicast could be used to implement the Registry.

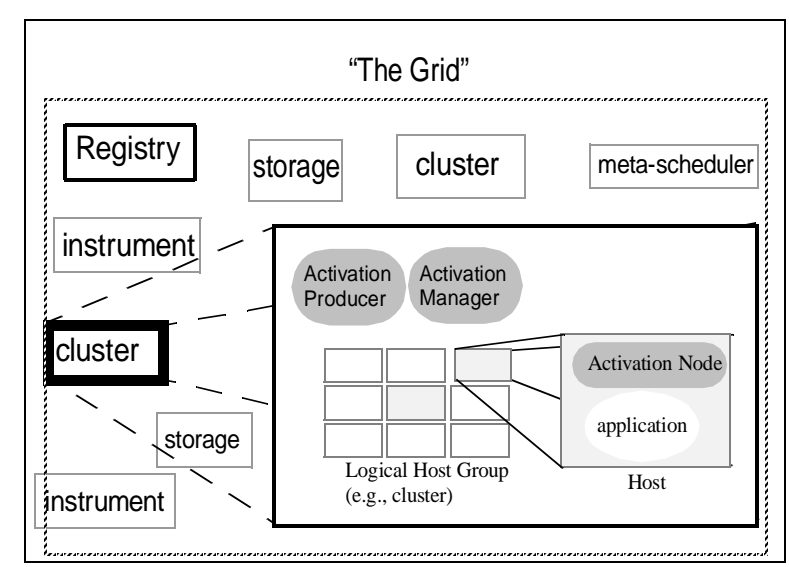

Figure 3: Activation Service 


\subsection{Activation Node}

The Activation Node is responsible for getting the desired logging level from the Activation Manager and communicating this level to the appropriate NetLogger-instrumented programs. It is also responsible for forwarding the instrumentation and monitoring data from these programs to the Activation Producer.

Applications that wish to be activated must use the NetLogger trigger API, which causes NetLogger to automatically periodically check a "trigger" file for updates to the logging level or destination. In addition, the trigger API will create a small file describing the NetLoggerized application; the Activation Node scans for these files in order to figure out which applications are running on a host.

To get the user-specified logging level, the Activation Node polls the Activation Manager using the pyGMA "query" operation, matches the results with the list of known NetLogger-instrumented programs running on this host, and modifies the NetLogger "trigger" file accordingly. Although it would be possible for the Activation Node to tell applications to log directly to the Activation Producer, this may cause delays if the Activation Producer becomes overloaded. Therefore, the Activation Node always "triggers" logging to a temporary file on local disk, and forwards the monitoring data asynchronously to the Activation Producer.

\subsection{Activation Producer}

The Activation Producer receives pyGMA subscriptions from consumers. As mentioned above, it also receives NetLogger instrumentation data from the Activation Node. The main task of the Activation Producer, then, is to match incoming instrumentation data with the subscriptions. In order to do this efficiently, the monitoring data is multiplexed, demultiplexed, and filtered by a NetLogger "pipe", part of the standard NetLogger library. In addition to using the efficient NetLogger encoding, by performing these functions inside the NetLogger library we also minimize copying of the monitoring data. Subscriptions are transformed into NetLogger "filters", which are added to the pipe, as illustrated in Figure 4.

Rather than try to build or borrow an expressive but complex filter language such as [12], we devised a simple method that would handle the most common use cases. NetLogger filters operate on one item of monitoring data at a time, testing to see if that item matches any single expression. An expression is a list of (name, operator, value) tuples. For example, a query that matches all "Start" or "End" monitoring events for program "Athena" at a logging level less than or equal to two would be:

NL.EVNT="Start" and PROG="Athena" and LVL <= 2

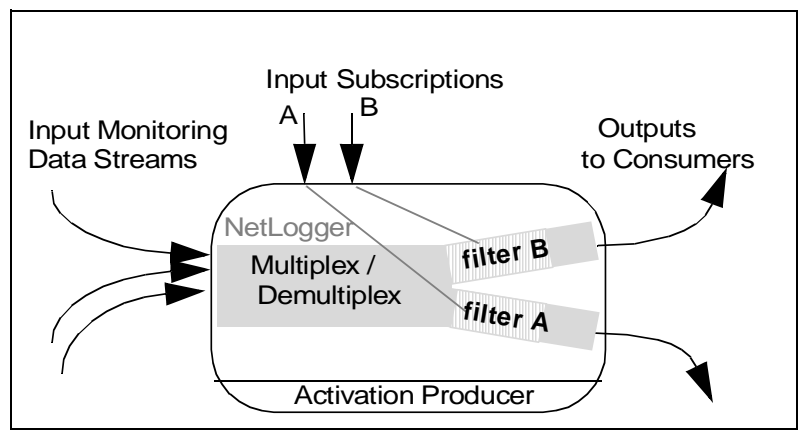

Figure 4: NetLogger "Pipe"

\section{NL.EVNT="End" and PROG="Athena" and LVL $<=2$}

This matches the NetLogger event:

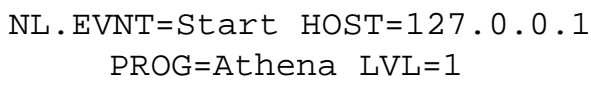

but does not match either of the NetLogger events:

$$
\begin{gathered}
\text { NL.EVNT=Middle HOST }=127.0 .0 .1 \\
\text { PROG=Athena LVL=1 } \\
\text { NL.EVNT=End HOST }=127.0 .0 .1 \\
\text { PROG=Athena LVL=3 }
\end{gathered}
$$

Due to the simplicity of the filter language, the implementation is straightforward and efficient. This is important because filtering is used extensively by the Activation Producer. Performance results for the NetLogger filtering API are shown in Section 5.2.

\subsection{Activation Manager}

The Activation Manager keeps track of the logging level for a given NetLogger-instrumented application. If the application is logging at level 3, then only log messages of level 0 through 3 will be produced; a logging level of -1 means "off". NetLogger instrumentation associates a log level with each piece of monitoring data, so an attempt to write monitoring data whose level is above the current logging level results in a no-op. This means that reducing the logging level is an easy and efficient way to reduce the overhead of instrumentation.

The Activation Manager is polled periodically by each Activation Node for its current list of "activations". One issue with this design is that with a large number of hosts (e.g., 500) and a small poll interval (e.g., 5 seconds), the request parsing can cause a high load on the Activation Manager host. This potential load is one reason that the architecture separates the Activation Manager from the Activation Producer.

If an Activation Manager or Activation Producer is overloaded, then these components can be replicated. Consumers will still be able to find the relevant components by searching the Registry (described below). Producers will either be pre-configured to find the right Activation Producer/Manager or may also search the Registry. 


\subsection{Registry}

Consumers who want to subscribe for monitoring data can search the Registry for the appropriate instance of the Activation Service. A typical search would be "find me the Activation Service associated with MyApplication on Cluster A or Cluster B". The Registry will return one or more Activation Service endpoints, and then the user can proceed to subscribe for the data, activate the logging, or both. The Registry could also be used to locate other GMA Producers with the same or related monitoring data, such as a monitoring data archive.

Because there is nothing about the Registry that is specific to the Activation Service, we have not yet attempted an implementation. Existing software, such as the MDS and R-GMA Registry, should serve admirably. It should be noted that we also have not needed a Registry up to this point, as all experiments have been run on a known cluster with a known associated Activation Service.

\section{Results}

In this section, we present results from using the Activation Service with an instrumented Athena Framework running on multiple nodes on a cluster. We focus on how the system scales as we increase the number of scheduled application instances (i.e.: number of cluster nodes used) and the number of "consumers" subscribing to the instrumentation data. Both simple and relatively complex subscriptions are employed, and a detailed (40 events/sec) logging level is activated. The next section describes the experimental setup, and subsequent sections present the results.

\subsection{Experimental Setup}

For the experiment, we ran Athena jobs on a queue in the NERSC "PDSF" cluster (http://pdsf.nersc.gov) in Oakland, CA, which has over 200 compute nodes available at any given time. The NetLogger instrumentation was sent to an Activation Producer at Lawrence Berkeley National Lab (LBNL) (about $15 \mathrm{~km}$ away), and the activation level was set and queried at an Activation Manager on the same subnet. Subscriptions (generated on a laptop) told the Activation Producer to send monitoring data to two remote hosts, one at Oak Ridge National Laboratory and one at the University of Pittsburgh Supercomputing Center. Note that we used remote hosts that had sufficient latency from the data source to emulate a realistic Grid environment. Five streams of monitoring data were sent to each consumer host. All hosts were $400 \mathrm{MHz}$ or higher Pentium systems running Linux; for more details see Appendix A.

In order to assess the performance of the entire system, various components were instrumented with NetLogger, and these logs were sent to yet another host on the LBNL subnet.
During each test, we measured the CPU load on the cluster nodes and on the Activation Producer and Activation Manager, and also the latency for each event between the time it was generated and the time it arrived at a Consumer.

\subsection{Filter Performance}

To better understand the performance characteristics of the Activation Service as a whole, we also tested the NetLogger "filter API" in isolation. Good performance here is crucial, as the filtering event rate provides an upper bound on the throughput of the Activation Producer. There are two independent variables which affect the event rate: filter complexity, and the proportion of events which 'pass' the filter. The more complex the filter, the longer it takes to evaluate each event. The more events which 'pass' the filter, the longer NetLogger spends performing I/O.

To evaluate the trade-off, we ran tests where the filter complexity varied from 0 to 40 comparisons in steps of 4 , and the proportion of events that 'passed' varied from $0 \%$ to $100 \%$ in steps of $10 \%$. The events used were similar to those in the Athena instrumentation. Results were logged from a host with the same configuration as hostB.lbl.gov, to the remote consumer in Pittsburgh (host.psc.edu).

Measuring the throughput versus the two independent variables produced the surface shown in Figure 5.

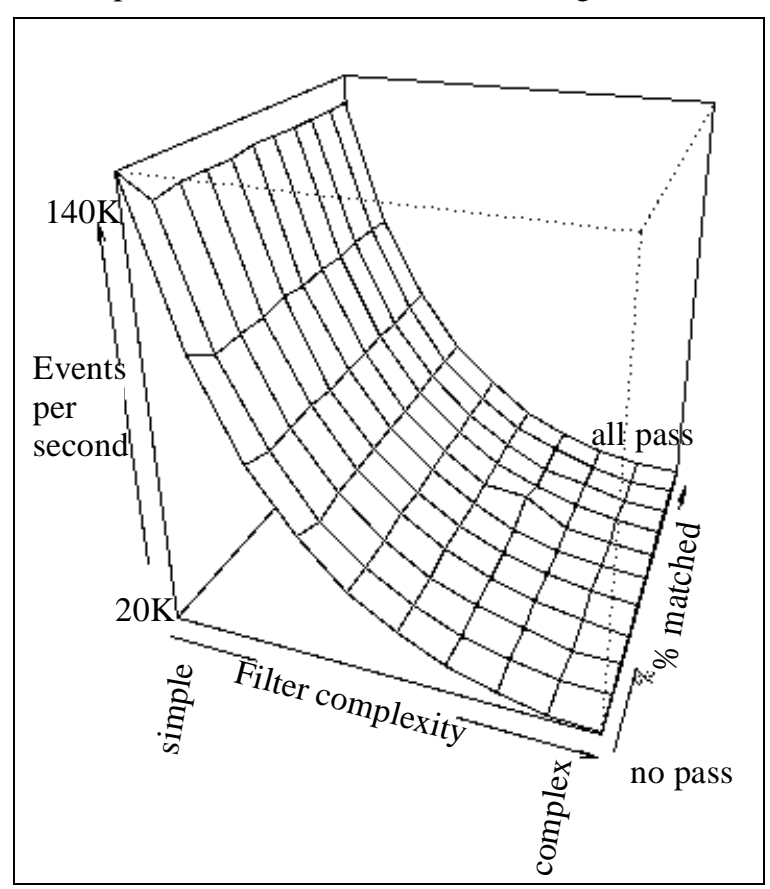

Figure 5: Filter Performance results

Clearly, the filter complexity is the main effect on performance. Between the simplest and most complex filters, there is roughly a factor of seven slow-down, whereas the $100 \%$ pass filter is only about five to ten percent slower than the $0 \%$ pass filter. 
However, even at reasonably high complexity, the filter performance is good. For example, when the filter has 20 expressions, on average it can write roughly 50,000 events per second. This is still faster than the raw speed of many less efficient logging libraries, such as $\log 4 j$ [7]. It should be noted that because the host used was relatively slow (400MHz) and filtering is CPU-intensive, most current hardware would have even better performance.

\subsection{Scalability}

The primary scalability question that we wished to address is: how many consumers and producers (instrumented jobs) can be handled by a single Activation Producer and Activation Manager? Secondarily, how does the complexity of the subscription affect these quantities? We measured the scalability of the system by comparing the average time for an event to travel from the instrumented job to the consumer as the number of producers, consumers, and event rate increased.

In our tests, we "pre-subscribed" to the Activation Producer with either a simple (4-comparison) or complex (20-comparison) filter expression, for each of 10 consumers. Then we submitted roughly 5-minute jobs to PDSF. We measured the event latencies for each event. The median values are graphed in Figure 6.

Before analyzing these results, some fixed sources of latency (particular to this implementation) should be mentioned. First, the forwarding of events from each instrumented job occurs in short bursts separated by 5 seconds. Second, each NetLogger Consumer and Producer uses buffers with a 1-second time-out to increase streaming efficiency. So, because each event is written, forwarded, read, written, and read again (see Figure 5) -the average fixed latency overhead is $((1+5+1+1+1) \div 2)=4.5$ seconds.

(Note that NetLogger provides a "flush" option to eliminate some of this latency, but that using it adds considerable I/O overhead, thus decreasing overall throughput.)

From 1 to 20 producers, the median event latency varied between 4.7 and 6.2 seconds. Neither consumer location or filter location seem to affect the values. Therefore, it seems that these latencies are random variation just above the fixed latency discussed above. This means that a single Activation Producer scaled to 20 producers at 40 events per second with a complex filter to

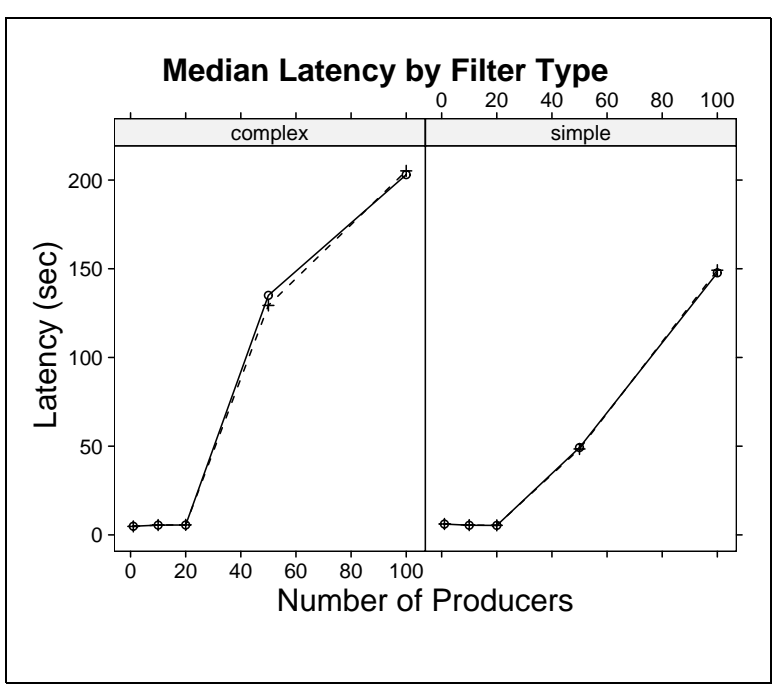

Figure 6: Scalability Test Results

10 consumers, i.e. an aggregate output rate of 8,000 events per second.

From 50 and 100 producers, we see linear increases in latency for both complex and simple filtering. As expected, the complex filter is slower than the simple filter. Again, consumer location does not affect latency.

In summary, the results show that the Activation Service scaled linearly with the product of the number of consumers and the number of producers. Very complex filters also added significantly to the load, however in all cases the performance was excellent with 20 producers and 10 consumers, and acceptable even with 100 producers and 10 consumers.

\section{Conclusions}

In this paper we described a Grid activation service which is designed to address the problem of starting, stopping, and changing the level of instrumentation data from running Grid processes. We have shown that a single Activation Producer node is efficient enough to handle an aggregate throughput of 8,000 monitoring events per second. In order to use the Activation Producer, the NetLogger instrumentation in Athena did not need to be modified at all, and only a single component (the Activation Node) needed to be added to the submitted job. The query language, although simple, provided sufficient flexibility for current needs. Overall, we believe that the Activation Services's flexible, distributed architecture will prove to be a useful building block for a comprehensive Grid monitoring and troubleshooting system.

\section{Acknowledgments}

This work was supported by the Director, Office of Science, Office of Advanced Scientific Computing Research. Mathematical, Information, and Computational Sciences Division under U.S. Department of Energy 
Contract No. DE-AC03-76SF00098 and by the Director, Office of Science, High Energy Physics, U.S. Department of Energy Contract No. DE-AC03-76SF00098. See the disclaimer at http://www-library.lbl.gov/disclaimer. This is report no. LBNL-52991.

\section{References}

[1] Athena Framework. http://atlas.web.cern.ch/ Atlas/GROUPS/SOFTWARE/OO/architecture/General/index.html

[2] M. Burger, T. Kielmann, H. Bal. TOPOMON: A Monitoring Tool for Grid Network Topology, International Conference on Computational Science (2), pp. 558-567, 2002.

[3] R. Byrom et. al., R-GMA: A Relational Grid Information and Monitoring System, Proceedings of the Cracow ' 02 Grid Workshop, January 2003. Web: https://edms.cern.ch/file/368364/1/rgma.pdf

[4] T. Dewitt, T. Gross, B. Lowekamp, N. Miller, P. Steenkist, J. Subhlok and D. Sutherland, ReMoS: A resource monitoring system for network aware applications, Tech. Rep. CMU-CS-97-194, School of Computer Science, Carnegie Mellon University, December 1997

[5] W. Feng, J. Hay, and M. Gardner, MAGNeT: Monitor for Application-Generated Network Traffic. 10th International Conference on Computer Communication and Networking. (IC $\left.{ }^{3} N^{\prime} 01\right)$, Scottsdale, Arizona, October 2001.

[6] I. Foster, C. Kesselman, J. Nick, S. Tuecke. The Physiology of the Grid: An Open Grid Services Architecture for Distributed Systems Integration. Open Grid Service Infrastructure WG, Global Grid Forum, June 22, 2002.

[7] D. Gunter, B. Tierney, K. Jackson, J. Lee, M. Stoufer, Dynamic Monitoring of High-Performance Distributed Applications, Proceedings of the 11th IEEE Symposium on High Performance Distributed Computing, HPDC-11 11, July 2002.

[8] Hawkeye, A Monitoring and Management Tool for Distributed Systems. http://www.cs.wisc.edu/condor/hawkeye/

[9] J. Hollingsworth and B. Tierney, Instrumentation and Monitoring, in The Grid, Volume 2. Morgan Kaufman, 2003.

[10] Kalmady R. and B. Tierney, A Comparison of GSIFTP and RFIO on a WAN, Proceedings of Computers in High Energy Physics 2001 (CHEP 2001).

[11] B. Miller, M. Callaghan, J. Cargille, J. Hollingsworth, R. Irvin, K. Karavanic, K. Kunchithapadam and T. Newhall. The Paradyn Parallel Performance Measurement Tool, IEEE Computer 28, 11, pp.37-46 (November 1995). Special issue on performance evaluation tools for parallel and distributed computer systems.

[12] J. Pereira, F. Fabret, F. Llirbat, D. Shasha. Efficient Matching for Web-Based Publish/Subscribe Systems, Conference on Cooperative Information Systems, pp. $162-173,2000$

[13] pyGMA, http://www-didc.lbl.gov/pyGMA
[14] M. Rose, The Blocks Extensible Exchange Protocol Core, RFC 3080, March 2001.

[15] B. Tierney, R. Aydt, D. Gunter, W. Smith, V. Taylor, R. Wolski, M. Swany, A Grid Monitoring Service Architecture, Global Grid Forum White Paper, http://www-didc.lbl.gov/GridPerf/.

[16] B. Tierney and D. Gunter, NetLogger: A Toolkit for Distributed System Performance Tuning and Debugging, LBNL Tech Report LBNL-51276

[17] B. Tierney, W. Johnston, B. Crowley, G. Hoo, C. Brooks, D. Gunter, The NetLogger Methodology for High Performance Distributed Systems Performance Analysis, Proceeding of IEEE High Performance Distributed Computing, July 1998, LBNL-42611. http://www-didc.lbl.gov/NetLogger/

[18] S. Tuecke, K. Czajkowski, I. Foster, J. Frey, S. Graham, C. Kesselman. Grid Service Specification. Open Grid Service Infrastructure WG, Global Grid Forum, Draft 2 , $7 / 17 / 2002$. http://www.gridforum.org/ogsi-wg/drafts/draft-ggf-ogsi-gridservice-29_2003-04-05.pdf

[19] Widener, P., G. Eisenhauer, K. Schwan, and F. Bustamante, Open Metadata Formats: Efficient XML-Based Communication for High Performance Computing, Cluster Computing, 2002.

\section{Appendix A: Experimental Host Details}

The LBNL hosts were all $450 \mathrm{MHz}$ Pentium III with 256MB of RAM, running RedHat Linux 7.2 (2.4.9 kernel) and had Gigabit Ethernet external connectivity. The round trip time between these machines and PDSF, as estimated by ping, was roughly $3 \mathrm{~ms}$. The remote host at psc.edu was a dual-processor $1 \mathrm{GHz}$ Pentium III with $892 \mathrm{MB}$ if RAM, running RedHat Linux 7.2 (2.4.20 kernel), with high-speed $(\sim 400 \mathrm{Mb} / \mathrm{s})$ connectivity to the LBNL hosts. The round trip time between this machine and PDSF, as estimated by ping, was roughly $70 \mathrm{~ms}$.

The PDSF cluster is heterogeneous, but most nodes have one or two Pentium III processors between $600 \mathrm{MHz}$ and $1 \mathrm{GHz}$. 